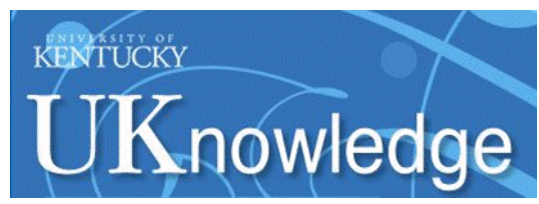

University of Kentucky

UKnowledge

\title{
Neurocognitive Basis of Repetition Deficits in Primary Progressive Aphasia
}

\author{
Sladjana Lukic \\ University of California, San Francisco \\ Maria Luisa Mandelli \\ University of California, San Francisco \\ Ariane Welch \\ University of California, San Francisco \\ Kesshi Jordan \\ University of California, San Francisco \\ Wendy Shwe \\ University of California, San Francisco
}

See next page for additional authors

Follow this and additional works at: https://uknowledge.uky.edu/csd_facpub

Part of the Communication Sciences and Disorders Commons

Right click to open a feedback form in a new tab to let us know how this document benefits you.

\section{Repository Citation}

Lukic, Sladjana; Mandelli, Maria Luisa; Welch, Ariane; Jordan, Kesshi; Shwe, Wendy; Neuhaus, John; Miller, Zachary; Hubbard, H. Isabel; Henry, Maya; Miller, Bruce L.; Dronkers, Nina F.; and Gorno-Tempini, Maria Luisa, "Neurocognitive Basis of Repetition Deficits in Primary Progressive Aphasia" (2019). Communication Sciences and Disorders Faculty Publications. 2.

https://uknowledge.uky.edu/csd_facpub/2

This Article is brought to you for free and open access by the Communication Sciences and Disorders at UKnowledge. It has been accepted for inclusion in Communication Sciences and Disorders Faculty Publications by an authorized administrator of UKnowledge. For more information, please contact UKnowledge@lsv.uky.edu. 


\section{Neurocognitive Basis of Repetition Deficits in Primary Progressive Aphasia}

Digital Object Identifier (DOI)

https://doi.org/10.1016/j.bandl.2019.04.003

Notes/Citation Information

Published in Brain and Language, v. 194.

(c) 2019 Elsevier Inc.

(c) 2019. This manuscript version is made available under the CC-BY-NC-ND 4.0 license

https://creativecommons.org/licenses/by-nc-nd/4.0/

\section{Authors}

Sladjana Lukic, Maria Luisa Mandelli, Ariane Welch, Kesshi Jordan, Wendy Shwe, John Neuhaus, Zachary Miller, H. Isabel Hubbard, Maya Henry, Bruce L. Miller, Nina F. Dronkers, and Maria Luisa Gorno-Tempini 


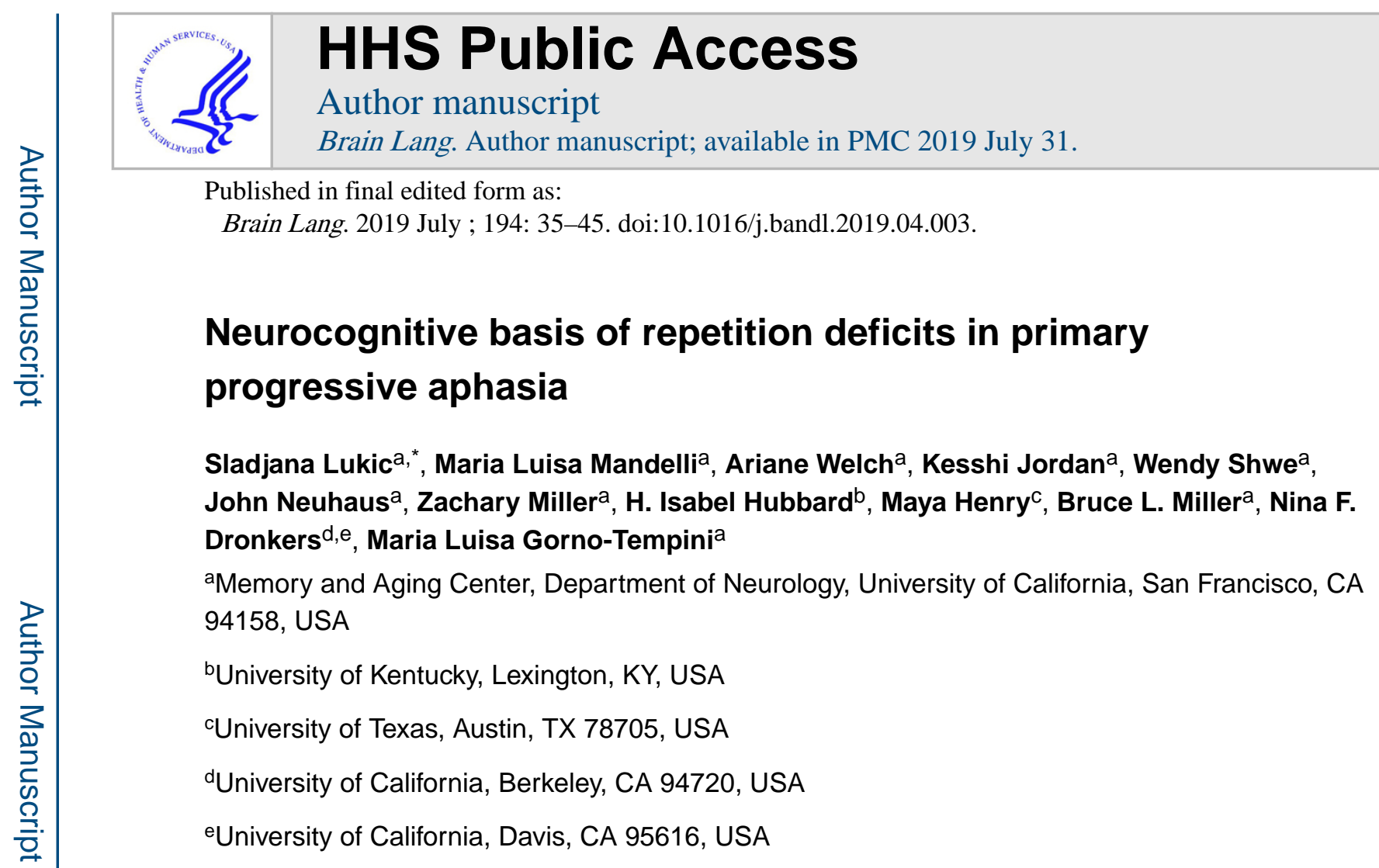

\begin{abstract}
Previous studies indicate that repetition is affected in primary progressive aphasia (PPA), particularly in the logopenic variant, due to limited auditory-verbal short-term memory (avSTM). We tested repetition of phrases varied by length (short, long) and meaning (meaningful, nonmeaningful) in 58 participants (22 logopenic, 19 nonfluent, and 17 semantic variants) and 21 healthy controls using a modified Bayles repetition test. We evaluated the relation between cortical thickness and repetition performance and whether sub-scores could discriminate PPA variants.

Logopenic participants showed impaired repetition across all phrases, specifically in repeating long phrases and any phrases that were non-meaningful. Nonfluent, semantic, and healthy control participants only had difficulty repeating long, non-meaningful phrases. Poor repetition of long phrases was associated with cortical thinning in left temporo-parietal areas across all variants, highlighting the importance of these areas in avSTM. Finally, Bayles repetition phrases can assist classification in PPA, discriminating logopenic from nonfluent/semantic participants with $89 \%$ accuracy.
\end{abstract}

\title{
Keywords
}

Cortical thickness; Phrase repetition; Length; Semantics; Primary progressive aphasia

\footnotetext{
*Corresponding author at: Memory and Aging Center, Department of Neurology, University of California San Francisco, 675 Nelson Rising Lane, Suite 190, San Francisco, CA 94158, USA. Sladjana.lukic@ucsf.edu (S. Lukic).

Appendix C. Supplementary material

Supplementary data to this article can be found online at https://doi.org/10.1016/j.band1.2019.04.003.

${ }^{1}$ Following Bayles et al. (1996), post hoc analyses were also done using the same data analysis procedures and substituting words used as the unit of measure. However, no differences in the results were obtained when coding for syllables versus words.
} 


\section{Introduction}

The neurocognitive basis of language repetition has been intensively studied in patients with stroke or, more recently, with primary progressive aphasia (PPA) (e.g., Baldo, Klostermann, \& Dronkers, 2008; Buchsbaum et al., 2011; Gorno-Tempini et al., 2008; Leyton et al., 2014). Previous studies used repetition performance to distinguish the three PPA variants: nonfluent/agrammatic (nfvPPA), semantic (svPPA), and logopenic (lvPPA) (Bonner, Ash, \& Grossman, 2010; Gorno-Tempini et al., 2011; Mesulam, 1982; Mesulam, Wieneke, Thompson, Rogalski, \& Weintraub, 2012; Weintraub, Rubin, \& Mesulam, 1990). For instance, poor repetition performance is associated with lvPPA (e.g., Gorno-Tempini et al., 2004, 2008), but also with nfvPPA (e.g., Leyton et al., 2014). Furthermore, imaging studies in patients and healthy participants have implicated inferior parietal and frontal cortices in repetition processes (see Majerus, 2013).

Repetition is a complex, multilevel process that taxes auditory-verbal short-term memory (avSTM). avSTM involves the short-term stores of phonological and semantic information, as well as articulatory rehearsal, both of which are necessary for maintenance and execution of verbal information (Baddeley, 2000, 2003). The short-term stores of phonological and semantic information are part of the phonological loop by which verbal information is temporarily stored and processed. These short-term stores can be refreshed by the execution of a subvocal articulatory "rehearsal" process (Baddeley, Hitch, \& Allen, 2009). Several neurocognitive models of language repetition have been proposed (Jacquemot \& Scott, 2006; Hickok \& Poeppel, 2007; Friederici, 2012; Hickok, 2009, 2012; also see Majerus, 2013 for a review). These models propose that in the dorsal stream of speech processing, the posterior superior temporal gyrus (pSTG) serves as a sensorimotor interface that links acoustic and phonological representations in the STG to articulatory representations and/or rehearsal in the posterior inferior frontal gyrus (pIFG). The phonological short-term maintenance function has a distinct set of neural substrates, including left inferior parietal areas such as supramarginal and angular gyri (SMG/AG).

In the area of stroke research, repetition deficits are considered a hallmark of conduction aphasia. This disorder originally was referred to as a "disconnection syndrome" relating to damage to a prominent white matter tract, the arcuate fasciculus (AF) (e.g., Geldmacher, Quigg, \& Elias, 2007; Tanabe et al., 1987; Yamada et al., 2007). However, others have indicated that damage to the AF is not a prerequisite for conduction aphasia (Baldo, Katseff, $\&$ Dronkers, 2012; Dronkers, 2000) and that patients with AF lesions may retain the ability to repeat words and phrases (Kreisler et al., 2000; Shuren et al., 1995). Patients with a form of conduction aphasia most often present with cortical lesions to the left STG and/or SMG, and exhibit deficits in repetition and speech production (Axer, von Keyserlingk, Berks, \& von Keyserlingk, 2001; Baldo \& Dronkers, 2006; Damasio \& Damasio, 1980; Green \& Howes, 1977). These patients have difficulty maintaining verbal information and most often produce phonological speech errors, which points to deficits in avSTM (Baldo \& Dronkers, 2006; Baldo et al., 2008; Buchsbaum et al., 2011). Furthermore, researchers argue for theoretically separable functional deficits: an inability to retain ("repetition") and an inability to produce ("reproduction") verbal units, with the former involving avSTM and the latter involving speech production process (see Shallice \& Warrington, 1977). In an 
extensive review, Buchsbaum et al. (2011) compared activation patterns in previously published fMRI studies of phonological STM with the lesion distribution of conduction aphasia. They compared a conjunction analysis of 105 single subject fMRI scans from a phonological STM paradigm with the lesion distribution of 14 patients with conduction aphasia. The results indicated that the areas of greatest overlap between fMRI activations and lesion locations were in the left posterior portion of the planum temporale, area Spt (Sylvianparietal-temporal), a site which has been argued to support sensory-motor integration for vocal tract actions (Hickok, Okada, \& Serences, 2009).

Patients with PPA, particularly the logopenic variant (lvPPA), have provided evidence that posterior temporo-parietal areas support sensory-motor integration and maintenance of phonological information during repetition. Voxel-based morphometry studies reveal that lvPPA is characterized by damage to the left posterior temporal and inferior parietal grey matter (Gorno-Tempini et al., 2004, 2008; Henry \& Gorno-Tempini, 2010; Hodges \& Patterson, 1996; Neary et al., 1998; Mesulam et al., 2009). Furthermore, MRI tractography studies show greatest white matter changes in the temporo-parietal component of left superior longitudinal fasciculus (SLF) that connects these regions, but also reveal abnormalities in the left arcuate fasciculus, in the frontoangular (SLF-II) and the frontosupramarginal (SLF-III), and in the right temporoparietal SLF (Galantucci et al., 2011).

Behaviorally, using both experimental tasks and standardized repetition tests, past research demonstrates that repetition is impaired in both lvPPA and nfvPPA (e.g., Gorno-Tempini et al., 2004, 2008; Henry \& Gorno-Tempini, 2010; Leyton et al., 2014; Rohrer et al., 2010; Whitwell et al., 2015). Subsequently, different theoretical accounts of repetition impairments in the different variants of PPA are proposed: reduced capacity of the phonological store in lvPPA (e.g., Gorno-Tempini et al., 2008; Meyer, Snider, Campbell, \& Friedman, 2015), and/or disrupted articulatory planning or rehearsal of encoded verbal information in nfvPPA (e.g., Gorno-Tempini et al., 2004; Ogar, Dronkers, Brambati, Miller, \& Gorno-Tempini, 2007; see also Martin, 1987, Martin, Shelton, \& Yaffee, 1994 on stroke patients). Moreover, the inability to repeat phrases or sentences may also arise from loss of semantic memory in svPPA (e.g., Hodges \& Miller, 2001; Knott, Patterson, \& Hodges, 1997; McCarthy \& Warrington, 1987).

Repetition abilities in healthy individuals are sensitive to factors such as word length and phonological similarity, with longer words (e.g., 1-5 syllable words) and similarly sounding words (e.g., 'mad', 'map') being more difficult to recall (Baddeley, Thomson, \& Buchanan, 1975; Conrad \& Hull, 1964). These effects are classically associated with normal functioning of the phonological store. Gorno-Tempini et al. (2008) tested these effects in 7 patients with lvPPA using digit, letter (similar and dissimilar), and word span tasks, as well as the Western Aphasia Battery (WAB) repetition subtest. These lvPPA patients showed normal repetition of single digits or letters, but were severely impaired in sequences of more than three digits or letters, and showed no phonological similarity effect. On the word span task, these patients could repeat three short words and only one long word, and were severely impaired on the WAB sentence repetition subtest. These findings supported their hypothesis that reduced phonological store might be the core deficit in lvPPA. Similarly, 
Leyton et al. (2014) tested verbal repetition in 63 patients (with variants of PPA and Alzheimer's Disease (AD)) and 13 healthy controls (HC) using several experimental tasks. They demonstrate impaired verbal repetition across groups: (1) the svPPA patients were impaired only in a sentence repetition task, (2) nfvPPA patients were impaired mostly in word repetition and digit span tasks, and showed phonological similarity effects on a word span task, (3) lvPPA patients were impaired in all tasks, with the most severe impairments in the sentence repetition and word span task, and showed a reduced phonological similarity effect, and (4) the AD group was impaired in span tasks and sentence repetition.

Past research in stroke and $\mathrm{AD}$ has explained the complex relationship between repetition deficits and memory resources by attributing difficulties in sentence repetition to reduced avSTM or semantic memory (e.g., Baldo and Dronkers, 2006, 2008; Knott et al., 1997). For instance, Baldo and Dronkers $(2006,2008)$ showed that patients with conduction aphasia have deficits in repetition recognition tasks, but only when the distractor sentences are semantically related to the target sentence. In contrast, patients are able to identify the matching sentence when the distractors are semantically distinct, suggesting that these patients rely on non-phonologic cues, such as lexical-semantics, to support their avSTM. Bayles, Tomoeda, and Rein (1996) tested the semantic memory loss theory by examining the ability of patients with $\mathrm{AD}$ to repeat phrases varying in length and meaning. A group of mild and moderate AD patients (57) and HC subjects (62) were given six- and nine-syllable phrases that were meaningful, improbable in meaning, or meaningless. The authors showed that both $\mathrm{AD}$ patients and $\mathrm{HC}$ subjects had the most difficulty repeating meaningless ninesyllable phrases (e.g., "hairpins leap fluttering riddle games"), which failed to confirm a performance pattern consistent with a semantic memory loss theory.

Generally, these results demonstrate that repetition ability is affected when the sequence of words is long and has no real meaning. Thus, both phonological and semantic (meaning) information contribute to sentence recall performance (see Meltzer et al., 2016 on the semantic contribution to short- and long-term sentence recall). Although researchers have studied the ability of patients with PPA to repeat words and sentences, none have systematically varied length and meaningfulness of stimuli. Similarly, most PPA repetition studies have observed repetition deficits across the three variants by using a small number of stimuli (5 words, 4 phrases and 6 sentences in the WAB repetition subtest) to enable direct comparisons. Using the total score of the widely used WAB repetition subtest across variants has precluded an examination of whether specific repetition deficits may assist in the classification of PPA into variants, and identification of shared or distinct neurocognitive deficits across these variants.

In the present study, we examined the effects of length (long vs. short phrases) and meaning (meaningful vs. non-meaningful phrases), and frequently-used sentences on repetition performance in patients with PPA and HC subjects using a shortened form of a repetition test by Bayles et al. (1996). We then used structural neuroimaging to evaluate the relationship between cortical thickness and repetition performances across PPA. Lastly, we used Receiver Operating Characteristic (ROC) Curve to evaluate whether different phrase subscores on the Bayles test can distinguish lvPPA from nfvPPA and svPPA. 
Specifically, we asked (1) whether there are performance differences between different repetition conditions (frequent sentences, and short and long meaningful and non-

meaningful phrases) for the given group (the within-group analysis), and (2) whether lvPPA patients have more difficulty with long and/or non-meaningful phrases (compared to short and meaningful phrases) than nfvPPA patients, svPPA patients, and HCs (the between-group analysis). We also extended these analyses by asking (3) whether distinct neural correlates are associated with repetition performances on each phrase condition across PPAs, and (4) whether the sub-scores from each phrase condition can discriminate lvPPA from nfvPPA and svPPA.

We anticipated that the lvPPA group would show poor repetition across all phrases on the Bayles repetition test, while svPPA patients, nfvPPA patients and HCs would show no repetition impairment overall, except for lower performance on long non-meaningful phrases, in line with the original study of AD by Bayles et al. (1996). Based on the auditoryverbal short-term memory account, we expected to find length and meaning effects, specifically that repetition difficulty would increase with phrase length, and also when the verbatim information has been lost to lvPPA patients. Like patients with conduction aphasia, lvPPA patients are expected to rely on the lexical-semantics of the phrase in order to repeat it due to a degraded phonological trace (in line with Baldo et al. $(2006,2008,2012)$ ). We predicted that these repetition deficits would be associated with atrophy in left temporoparietal areas. Finally, we expected that the repetition subscores would distinguish lvPPA from the other PPA groups, suggesting that these subscores may be a useful tool in diagnostic assessment.

\section{Methods}

\subsection{Participants}

Fifty-eight patients with primary progressive aphasia (PPA), and twenty-one age- and education-matched healthy controls (HC) took part in this study. All participants were recruited through the Memory and Aging Center (MAC) at the University of California San Francisco (UCSF). All participants provided written informed consent approved by the UCSF Institutional Review Board. The fifty-eight patients (36 females, 8 left-handed/ ambidextrous, mean $(\mathrm{SD})$ age $=64( \pm 7)$ years, mean $(\mathrm{SD})$ education $=17( \pm 2.9)$ years $)$ were diagnosed with PPA according to proposed consensus clinical criteria (Gorno-Tempini et al., 2011), and, hence, classified into one of three PPA variants: logopenic (lvPPA $=22)$, non-fluent/agrammatic (nfvPPA $=19)$, and semantic $(\mathrm{svPPA}=17)$.

Diagnosis consensus was reached based on the comprehensive multidisciplinary evaluation including neurological history and examination, neuropsychological testing, and neuroimaging, as previously described (Gorno-Tempini et al., 2004). PPA participants selection criteria were: a score 11 or greater on the Mini-Mental State Examination (MMSE; Folstein, Folstein, \& McHugh, 1975), an assessment of repetition performance, and the availability of an MRI scan. Coincidentally, this selection led to a larger number of lefthanded/ambidextrous lvPPA patients than would be expected in the general population or a larger cohort of lvPPAs. LvPPA patients presented with impaired repetition on the repetition subtest of Western Aphasia Battery (WAB; Kertesz, 1982), and had a low digit span 
backwards, whilst having spared single word comprehension, motor speech, and grammar. SvPPA patients showed single word naming and comprehension deficits as evaluated with pictures from the Boston Naming Battery (BNT; Goodglass, Kaplan, \& Weintraub, 1983) and Peabody Picture Vocabulary Test (PPVT-Revised, Dunn \& Dunn, 1981), respectively. Both lvPPA and svPPA patients had low semantic (animal) fluency. Finally, nfvPPA patients presented with significant impairments in speech fluency on the spontaneous speech section from the WAB, and the severity ratings of apraxia of speech. Also, structural neuroimaging (MRI) was conducted for all PPA participants but was not considered for diagnosis (see Appendix A for cortical atrophy patterns for the three PPA variants).

All HC ( $\mathrm{n}=21 ; 15$ females, 8 left-handed, mean $(\mathrm{SD})$ age $=67( \pm 6)$ years, mean $(\mathrm{SD})$ education $=17( \pm 1.4)$ years) performed within normal limits on neuropsychological and speech-language tests, and had no history of speech-language, neurological disorders, or psychiatric disturbances (self-reported and screened). Left-handed controls (8/21) were recruited in order to match for handedness in the patient group (8/58). Demographics, speech-language, and cognitive scores, and expected significant group differences are provided in Table 1.

\subsection{Repetition measure}

Participants completed a shorter Bayles repetition test, which was comprised of 20 phrases that varied in length and meaning. The test included four conditions: (1) short meaningful (SM: Rare poisonous lizard) and non-meaningful (SNM: Crawling summer trombone) phrases, and (2) long meaningful (LM: Antique furniture draws good patrons) and nonmeaningful (LNM: Hairpins leap fluttering riddle games) phrases. There were 5 phrases per condition. Five frequently-used sentences (FS: That must have cost a pretty penny) were also included as a control condition. The meaningful phrases were defined as truthful, logical, and likely expressions, whereas the non-meaningful phrases were defined as not logically possible. The short phrases contained six syllables (three words) and mainly were comprised of a noun as the head-word and two premodifiers complementing the head (e.g., "rare poisonous lizard'). The long phrases contained nine syllables (three- to five-word sequences) in which a verb functioned as the head, preceded or followed by noun phrases or an adverb (e.g., "antique furniture draws good patrons").

The word-form frequency (the total number of tokens for a given word) was calculated for each word within each phrase based on the Corpus of Contemporary American English (COCA; Davies, 2008). The average frequency of each multi-word phrase was generated for each condition. While no significant difference was found between meaningful and nonmeaningful conditions $(p=0.55)$, the long and short conditions could not be matched on average multi-word frequency $(p=0.002)$ as the long phrases tended to occur with higher frequency $(M=5.20, S D=0.31)$ than the short phrases $(M=4.68, S D=0.34)$. In addition, Bayles et al. (1996) reported that all phrases were words with standard frequency indices ranging from 25.10 to 72.70 and there were no statistical differences between the four conditions. Appendix B displays a complete list of phrases (see also Bayles et al. (1996) for the complete list of original phrases and their characteristics). 
Participants were instructed to repeat each phrase and received two points for each correctlyrepeated syllable. Points were deducted for phonological errors such as substitutions or additions of phonemic segments, or deletions of phonemes/whole syllables. However, articulatory errors (lack of accuracy or clarity in speech sounds) were not penalized when the word was clearly intelligible. Each 6-syllable phrase was worth 6 points and each 9syllable phrase was worth 9 points, so participants could score a total of 30 or 45 points depending on the condition.

\subsection{Data analysis}

For the repetition test, accuracy (correct/incorrect) was recorded for each syllable. ${ }^{1}$ Means and standard deviations were calculated for each word, producing the total percent correct of repeated syllables for each subject in each condition. The two primary questions were: (1) whether there would be performance differences on different repetition conditions within each given group (the within-group analysis), and (2) whether the lvPPA patients would have more difficulty with long and non-meaningful phrases (compared to short and meaningful phrases) than nfvPPA and svPPA patients, and HC (the between-group analysis).

The repetition accuracy on the within-group factor Condition (levels: FS, SM, SNM, LM, and LNM) was analyzed using general linear models with the appropriate random effect structure (i.e., random by-participant intercepts) as well as Tukey's honest significant difference (HSD) for the planned posthoc comparisons. For the between-group analysis, the repetition accuracy was analyzed using general linear models, with mixed-effects: fixed effects for Length (short, long) and Meaning (meaningful, non-meaningful), and the appropriate random effect structure (i.e., random by-participant intercepts). Length, meaning, and group effects, and two-way interactions between group and length or meaning of phrases were examined. Age, handedness, gender, and severity (measured by the CDR Box Score) were entered as covariates in each regression model. Regression was performed using the Ime4 package running in R program (http://www.r-project.org). Statistical significance was determined based on the 0.05 threshold. The adjusted $p$ values were calculated using Tukey's posthoc as implemented in the lsmeans package (https://cran.rproject.org/package=lsmeans).

\subsection{Neuroimaging}

A 3T Trio (Siemens) scanner was used to obtain structural 3D T1-weighted images at UCSF. The T1-weighted images were acquired using an MP-RAGE sequence with the following parameters: repetition time $(\mathrm{TR})=2300 \mathrm{~ms}$, echo time $(\mathrm{TE})=2.98 \mathrm{~ms}$, inversion time $=$ $900 \mathrm{~ms}$, flip angle 9 degrees, matrix size $=256 \times 240$, voxel size $1 \mathrm{~mm}$ cubed isotropic. Neuroimaging data pre-processing and statistical analyses were performed using the Computational Anatomy Toolbox (CAT12; http://dbm.neuro.uni-jena.de/cat) in Statistical Parametric Mapping software (SPM12; http://www.fil.ion.ucl.ac.uk/spm/software/spm12) under Matlab 2017 (http://www.mathworks.com). The T1-weighted images were bias-field corrected, skull-stripped, aligned to the Montreal Neurological Institute (MNI) standard

Conflict of interest

The authors declare no conflicts of interest regarding publication of this paper. 
space, and segmented into gray matter (GM), white matter (WM) and cerebrospinal fluid (CSF). Cortical thickness was measured with the Projection-based Thickness Method as described in Dahnke, Yotter, and Gaser (2013). This method uses tissue segmentation to estimate the WM distance, then projects to the local maxima (which is equal to the cortical thickness) to other GM voxels using a neighbor relationship. This method accounts for partial volume information, sulcal blurring, and sulcal asymmetry. The local maps were resampled and smoothed using a 15-mm Gaussian heat kernel (Yotter, Nenadic, Ziegler, Thompson, \& Gaser, 2011).

The surface-based morphometry (SBM) statistical analysis was conducted to examine the association between cortical thickness and repetition performance across PPA groups using multiple (linear) regressions. Specifically, we examined whether there were atrophic regions associated with repetition performance on all phrases, and whether these areas were affected when longer or non-meaningful phrases were involved as opposed to when short or meaningful phrases were involved. Age, handedness, gender, and severity were entered as covariates in each regression model. A significance threshold at $p<0.05$ family-wise error (FWE) cluster-level correction was used to detect areas of peak cortical thinning (atrophy) associated with repetition deficits across PPA groups using the threshold-Free Cluster Enhancement function in CAT12.

\subsection{Receiver Operating Characteristic (ROC) curve analysis for diagnostic repetition test}

We also investigated whether certain phrases in the Bayles test could (1) differentiate lvPPAs from the other (non-logopenic) patients, and (2) distinguish lvPPA from nfvPPA. Thus, we computed the ROC curves displaying the sensitivity and specificity of the repetition test at varying thresholds by comparing the subscores in the different repetition conditions for the lvPPA patients with non-logopenic patients. The true positive rate (sensitivity) on the y-axes denotes the fraction of patients who are correctly identified as logopenic with repetition test subscores. The false positive rate $(1-$ specificity) on the x-axes denotes the fraction of patients who are falsely identified as logopenic with repetition test subscores. The subscores from each condition were coded with a binary value of 0 or 1 and were used to compute the ROC curves across varying thresholds and the area under the curve (AUC) calculated. The AUC represents the probability that a randomly selected logopenic patient will have a lower test score than a randomly selected non-logopenic patient. The AUC of each subscore was then evaluated on the basis of the corresponding $95 \%$ confidence interval (CI) according to 1000 bootstrap replications. A perfect test would have an ROC curve going straight up to and over from the top left corner (100\% sensitivity, 100\% specificity) with an AUC of 1.

\section{Results}

\subsection{Repetition data}

Within-group linear regression results-Data from the repetition test were first analyzed within each group to evaluate the pattern of performance in the repetition conditions (frequent sentences and short and long meaningful and non-meaningful phrases). The dependent variable was accuracy calculated as the proportion of syllables repeated correctly. The HC, nfvPPA, and svPPA participants were highly accurate in their overall 
performance on the repetition test with a mean accuracy of $99 \% \pm 2.9 \%, 92.4 \% \pm 12.8 \%$, $91.7 \% \pm 13.9 \%$, respectively. Like HC, nfvPA and svPPA participants were less accurate in repeating long non-meaningful phrases compared to all other conditions $(p<0.001)$.

In contrast, lvPPA was less accurate overall $(65.9 \% \pm 31.4 \%)$ and performed increasingly worse as length increased. A Tukey post hoc comparison revealed that the lvPPA group's performance was significantly less accurate on long non-meaningful phrases compared to all other conditions $(p<0.001)$, and also significantly less accurate on long meaningful phrases compared to all other conditions $(p<0.001)$. In addition, the lvPPA group was less accurate in repeating short non-meaningful phrases compared to frequent sentences $(p=0.004)$. There was no significant performance difference between the short non-meaningful and meaningful phrases $(p=0.828)$, and short meaningful phrases and frequent sentences $(p=$ 0.082 ). Accuracy means and standard deviations for each condition on the repetition test are summarized in Table 2. Fig. 1 illustrates regression analysis results across conditions within each group.

Between-group linear regression results-In the between-group analyses, the linear mixed-effects regression analyses indicated that repetition accuracy was significantly predicted by group, length, and meaning. The HC group repeated significantly better compared to lvPPA $(t(111.04)=10.981, p<0.001), \operatorname{nfvPPA}(t(103.14)=4.915, p<0.001)$, and svPPA groups $(t(90.21)=4.727, p<0.001)$. However, lvPPA patients repeated significantly less accurately compared to nfvPPA $(t(148.66)=8.078, p<0.001)$ and svPPA $(t(158.18)=7.685, p<0.001)$ participants, while there was no difference in repetition performance between nfvPPA and svPPA groups $(t(171.55)=0.325, p=0.746)$. Both HC and PPA participants' repetition of long phrases was less accurate than short phrases $(t$ $(233.00)=15.558, p<0.001)$, and repetition of non-meaningful phrases was less accurate than meaningful phrases $(t(233.00)=-3.164, p=0.002)$.

To determine whether the group effect was driven by length or meaning of the phrases, we included two-way interactions in the regression models. A two-way interaction was significant between group and length of phrases, indicating that the pattern of performance in the different length conditions was different for the three PPA groups and HC. The magnitude of the difference between long and short phrases was larger for lvPPA than for the HC $(t(233.00)=-10.301, p<0.000), \operatorname{nfvPPA}(t(233.00)=-6.728, p<0.000)$ and $\operatorname{svPPA}(t(233.00)=-5.910, p<0.000)$. Also, the group difference for long phrases was more distinct compared to the group difference for short phrases. In contrast, the two-way interaction was not significant between group and meaning of phrases, which indicates that the pattern of performance in the two meaning conditions was not different for the three PPA groups and HC. Fig. 2 illustrates the between-group regression analyses results across conditions for the three PPA groups and the group of HC.

\subsection{Cortical thickness and repetition performance correlations across PPAs}

Imaging analysis using surface-based morphometry (SBM) revealed that the repetition performance on all phrases was positively associated with cortical thickness in the superior temporal gyrus (STG) and supramarginal/angular gyri (SMG/AG). The Long phrases versus 
Short phrases revealed a cluster of significant thinning in the left STG, SMG, and AG.

However, for Short phrases versus Long phrases, no regions survived the cluster-correction for significance. Similarly, the Non-meaningful versus Meaningful phrases and the opposite contrast Meaningful versus Non-meaningful phrases revealed no significant areas of thinning. Table 3 summarizes the areas where cortical thinning was significantly associated with performance on overall repetition and long phrases. Fig. 3 displays cortical thickness maps showing areas where cortical thinning was associated with the overall repetition performance, and with long over short phrases across PPAs.

\subsection{Sensitivity and specificity of different repetition subscores for identifying the IvPPA patients}

The revised Bayles test subscores were evaluated as a metric for discriminating lvPPA from the other (non-logopenic) patients. Therefore, the AUC was generated for various condition sub-scores on the Bayles test. The LM and LNM phrases had the AUC [CI] of 0.90 [0.80, $0.97]$ and 0.91 [0.82, 0.98], respectively, which indicates that the subscores from the LM and LNM are equally good in classifying patient with logopenic PPA. In contrast, the FS, SM and SNM phrases had the AUC of 0.67 [0.54, 0.81], 0.74 [0.61, 0.86] and 0.79 [0.65, 0.91], respectively, which indicates that these phrases were much less important in characterizing the lvPPA and non-logopenic patients.

Similarly, we pursued the more relevant clinical question of whether these phrases on the Bayles test could distinguish lvPPA from nfvPPA. The LM and LNM phrases had the AUC $[\mathrm{CI}]$ of $0.91[0.79,0.98]$ and $0.91[0.81,0.98]$, respectively, which indicates that the subscores from the LM and LNM are equally good in classifying patient with logopenic PPA. In contrast, the FS, SM and SNM phrases had the AUC of 0.65 [0.48, 0.79], 0.72 [0.57, $0.86]$ and 0.80 [0.66, 0.92], respectively, which indicates that these phrases were much less important in characterizing the lvPPA and nfvPPA patients. Fig. 4 displays ROC curves showing the capacity of different repetition subscores to discriminate the lvPPA from the non-logopenic patients (4A) and nfvPPA patients (4B).

\section{Discussion}

The present study used a set of phrases varying in length and meaning to probe repetition processing in PPAs and HC subjects. Specifically, we tested the prediction that reduced auditory-verbal short-term memory (avSTM) would give rise to repetition errors whenever the verbal repetition process included longer and less meaningful/unfamiliar phrases and sentences. In addition, it was predicted that these errors would be associated with atrophy in the left temporo-parietal junction. The current findings generally supported these predictions. LvPPA patients were impaired in their overall ability to repeat phrases and showed impairments in repeating particularly long (over short) phrases, and were the only group that showed deficits in repeating short non-meaningful phrases. In contrast, nfvPPA and svPPA patients, similar to HCs, were highly accurate on the repetition task but less accurate in repeating long, non-meaningful phrases. Thickness in the left temporo-parietal areas positively correlated with performance on the repetition test across the three PPA subtypes. Last, we showed that long meaningful and non-meaningful phrase subscores 
discriminated lvPPA from the other (non-logopenic) patients and nfvPPA. These data suggest that avSTM is reduced in lvPPA patients, causing poor performance on the current modified Bayles test.

\subsection{Patterns of repetition impairments}

Most previous studies that have investigated repetition processing in PPAs rely on the available standardized repetition tests. Not many alternative validated tests of repetition are as comprehensive as the WAB repetition subtest. This test contains 15 stimuli of increasing difficulty including single words (1-5), phrases (6-9), and sentences (10-15). However, the first nine items are easy and all three PPA variants perform quite well; only the six most difficult sentences have the sensitivity necessary to capture repetition deficits in PPAs (Mesulam et al., 2012; but see also Table 1 in the current study). For example, Mesulam and colleagues showed poor sentence repetition in lvPPA patients but also showed such deficits to be prominent in nfvPPA patients. Theoretical accounts of these deficits suggest that inability to repeat words or sentences is driven by (1) limited verbal short-term memory, which is taxed by longer utterances, (2) loss of semantic memory or (3) rehearsal control errors, which produce failures in articulatory planning. Yet, this and other studies that employed the WAB repetition subtest were unable to differentiate the three repetition impairments, and to test effects of word, phrase or sentence length. The WAB subtest was not sufficiently sensitive to separate the PPA variants, especially in the early stages of the disease, as pointed out by Mesulam et al. (2012).

In our study, we examined the effects of length and meaning on phrase repetition using a between-group design, and found length and meaning effects, with PPAs being less accurate in repeating long (over short) and non-meaningful (over meaningful) phrases. Furthermore, there was a significant interaction effect between group and length of phrases. For the lvPPA group, the magnitude of difference between long and short phrases was larger compared to other groups. The two-way interaction was not significant between group and meaning of phrases, which indicates that the pattern of performance in the two meaning conditions was not different for the three PPA groups and HC. Specifically, we observed some common and distinct patterns across groups. Here, the repetition of long frequent sentences was highly accurate and the repetition of long non-meaningful phrases was the most inaccurate (in line with original findings by Bayles et al., 1996). In contrast to the other groups, lvPPA patients showed unique performance patterns: they were significantly worse in repeating long nonmeaningful and meaningful phrases, as well as short non-meaningful phrases. Moreover, lvPPA patients performed significantly worse on short non-meaningful phrases as compared to frequently-occurring sentences. In contrast to previous studies, these data reveal that sentences are not necessarily more difficult (less accurate) to repeat than single phrases. These patterns suggest that tasks requiring the repetition of both long and short phrases that cannot rely on semantic and/or syntactic information could be crucial in diagnosing lvPPA clinically.

The ROC results also demonstrated the ability of short non-meaningful phrases and long meaningful and non-meaningful phrases in discriminating lvPPA from non-logopenic patients and nfvPPA, suggesting that these subscores could be used in diagnostic 
assessments. Appendix B lists the seven phrases that best discriminate logopenic from nonlogopenic patients, based on a subjective cut-off ( $>85 \%$ of the total syllables repeated correctly) and the highest Area Under the Curve (AUC > 0.8). These findings are consistent with the conclusions of Baldo et al. $(2006,2008)$ that individuals with conduction aphasia and/or inferior parietal lesions are unable to retain the auditory trace of a sentence, and rely instead on its meaning. Thus, high-frequency sentences (e.g., "The telephone is ringing") can be repeated with high accuracy, but those that occur infrequently in conversation (e.g., "The pastry cook was elated") are not. In particular, these same patients often reply, "it was something about a happy baker" (Dronkers, Redfern, \& Ludy, 1998). In the present study, our control sentences were not only frequent, but also rather idiomatic, and thus were feasible for the lvPPA group to repeat in comparison to phrases that were longer and nonmeaningful. The short, meaningful phrases included a conjoined adjective and noun phrases (e.g., "remote tropical isle"), in which two words shared thematic and grammatical roles; this may have facilitated phrase repetition through easier access to phrase meaning.

\subsection{Anatomical correlates of repetition impairments}

Our findings are largely consistent with studies that have investigated neural substrates of repetition deficits in stroke and PPA. Previous studies on conduction aphasia in stroke have shown that lesions to the left temporal and parietal cortices are associated with repetition deficits (Baldo \& Dronkers, 2006; see Buchsbaum et al., 2011 for a review). The posterior temporo-parietal areas were also the most atrophied in patients with lvPPA who presented with core impairments in repetition (e.g., Gorno-Tempini et al., 2008; Meyer et al., 2015).

To build on this observation, we assessed the correlation between cortical thinning and repetition performance using whole-brain vertex thickness analyses across PPA groups, controlling for severity of the disease, and handedness. The left superior temporal (STG) and inferior parietal (SMG/AG) cortices were found to be involved in overall repetition performance. With respect to performance on longer phrases, clusters of significant thinning were found in the left lateralized STG and SMG.

These results are consistent with volumetric studies showing the distribution of atrophy in posterior perisylvian regions as well as altered white matter tracts in the temporoparietal component of the dorsal pathway (Gorno-Tempini et al., 2008; Hu et al., 2010; Galantucci et al., 2011; Rogalski et al., 2011). These results also are consistent with neurocognitive models of language repetition (Jacquemot \& Scott, 2006; Hickok \& Poeppel, 2007; Friederici, 2012; Hickok, 2012). According to these models, structures in left inferior parietal and temporal cortices (area Spt (Sylvian-parietal-temporal)) support the sensorymotor integration that is necessary for the maintenance of phonological information (Hickok \& Poeppel, 2007; Hickok, 2009; also see Majerus, 2013 for a review). These are avSTM components/processes that are common to our repetition measure.

During repetition, long-term phonological and semantic representations are activated and temporarily maintained via dorsal (pSTG/inferior parietal areas) and ventral (middle/inferior temporal cortex) repetition pathways, respectively (Majerus, 2013). Our lvPPA patients present with atrophy in dorsal regions and show repetition impairments, suggesting that difficulties in maintaining phonological information during repetition may at least partially 
explain the deficits observed in these patients. These results confirm the hypothesis that the primary anatomical/cognitive deficit in lvPPA patients is caused by damage to the pSTG and inferior parietal regions, resulting in the disruption of auditory short term verbal memory and perhaps linked to the interaction of sensory and motor systems.

To address the greater-than-expected rate of left-handed participants in our patient group, we performed a posthoc correlational analysis of the relationship between cortical thinning and repetition performance without covarying for nunisance variables, specifically handedness and severity (see supplementary material). The results of this posthoc analysis diverge from those obtaining controlling for the above mentioned variables, where significant correlations were found in the left temporo-parietal lobe. In the posthoc a more distributed network of areas is observed, including the left and right inferior parietal and superior temporal areas, as well as the left lateral occipital area. This may be influenced by the trend that left-handed patients presented with more symmetrical and bilateral atrophy. Given the statistical power granted by our sample, we can only assume that this difference is explained by some combination of handedness and/or severity, but cannot evaluate the contribution of each variable independently: when thickness values are correlated separately with severity and handedness, no significant results are found. The differences in results with and without these variables are indeed interesting and worthy of further study with a larger cohort of patients selected in a more systematic way. Future studies could properly investigate the contribution of the left and right hemispeheres to repetition performance, as well as the role of handedness and/or severity.

\section{Conclusion}

We utilized a modified version of the Bayles repetition to test the effects of two factors, length and meaning, on sentence repetition accuracy in three different groups of PPA patients. Repetition accuracy was best for short and meaningful phrases across all PPA groups. LvPPA patients showed a gradual decrease in repetition accuracy across length and meaningfulness conditions. Cortical thinning within the left temporo-parietal regions contributed to poor repetition accuracy across PPA groups, providing evidence that these regions are implicated in a brain network supporting avSTM. This study shows that specific neuroanatomical and linguistic features of the repetition test can inform the diagnosis of lvPPA. The insight from these findings may help distinguish clinically-relevant subprocesses, such as semantics or phonology, that underlie repetition deficits, which in turn has implications for models of avSTM, linguistic knowledge and semantic resources in neurodegenerative diseases. This present study provides key insight into poor patient performance on tasks that place particular demands on avSTM, including repetition and comprehension of spoken sentences and phrases. Given that these scores are a critical source of reliable classification of PPA into subtypes, these findings will increase the accuracy by which underlying pathology can be predicted from the clinical presentation in PPA.

\section{Supplementary Material}

Refer to Web version on PubMed Central for supplementary material. 


\section{Acknowledgments}

This work was supported by the National Institutes of Health (M.L.GT., NINDS R01 NS050915, NIDCD K24 DC015544; NIA U01 AG052943; M.H., R01DC016291-02; B.L.M., NIA P50 AG03006, NIA P50 AG023501, NIA P01 AG019724; N.D. NIDCD RO1 DC016345); State of California (M.L.GT., DHS04-35516); Alzheimer's Disease Center of California (B.L.M., 03-75271 DHS/ADP/ARCC); John Douglas French Alzheimer's Foundation (B.L.M.); Koret Family Foundation; The Bluefield Project to Cure Frontotemporal Dementia (B.L.M.); and McBean Family Foundation (B.L.M.). We thank our patients and healthy volunteers for participating in the research.

\section{Appendix A}

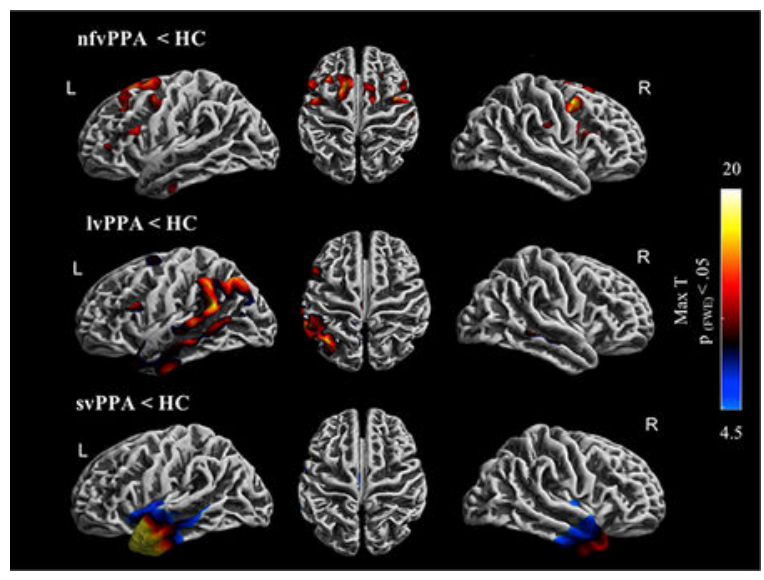

Cortical thickness maps showing atrophy patterns for PPA variants compared to HC. The presented map is thresholded at $\mathrm{p}<0.001$ family-wise error (FWE) corrected both at peaklevel and cluster extent based thresholding. Covariates: age, gender and handedness.

\section{Appendix B}

\begin{tabular}{ll}
\hline Conditions \\
\hline Frequent sentences \\
$1 \quad$ Is there something I can do for you? \\
$2 \quad$ That must have cost a pretty penny. \\
$3 \quad$ I don't know what you're talking about. \\
$4 \quad$ I am afraid I have some bad news. \\
$5 \quad$ Can I talk to you for a second? \\
Short meaningful phrases \\
$1 \quad$ Remote tropical isle \\
$2 \quad$ Cracked enamel surface \\
3 & Shaggy brown buffalo \\
4 & Rare poisonous lizard \\
5 & Foreign owned restaurant \\
Short non-meaningful phrases \\
1 & Crawling summer trombone \\
2 & Incapable top spoons
\end{tabular}




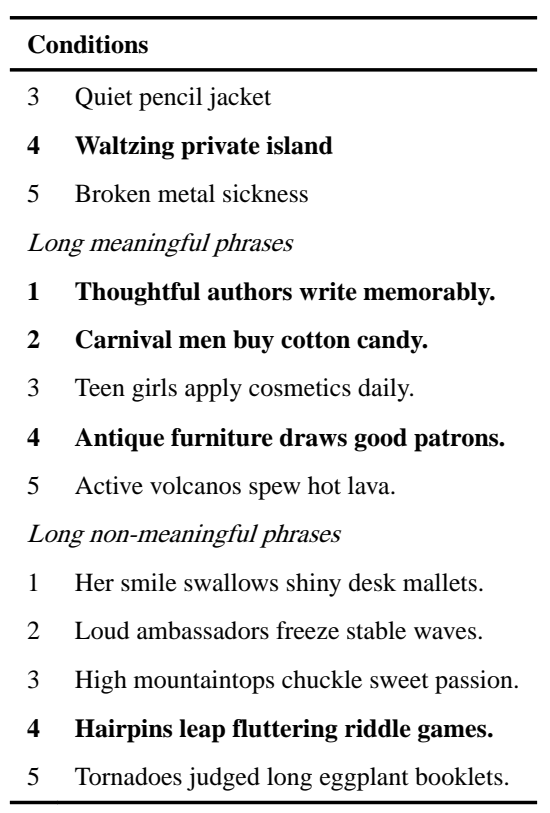

Note: The seven sentences in which lvPPA perform the most poorly and nfvPPA and svPPA perform well are in bold. This selection is based on a subjective cut-off $>85 \%$ of the total syllables repeated correctly, as well as the highest Area Under the Curve values (AUC >0.8).

\section{References}

Axer H, Keyserlingk AGV, Berks G, \& Keyserlingk DGV (2001). Supra-and infrasylvian conduction aphasia. Brain and Language, 76(3), 317-331. [PubMed: 11247647]

Baddeley AD, Thomson N, \& Buchanan M (1975). Word length and the structure of short-term memory. Journal of Verbal Learning and Verbal Behavior, 14(6), 575-589.

Baddeley AD (2000). The episodic buffer: A new component of working memory? Trends in Cognitive Sciences, 4(11), 417-423. [PubMed: 11058819]

Baddeley AD (2003). Working memory: Looking back and looking forward. Nature Reviews Neuroscience, 4(10), 829-839. [PubMed: 14523382]

Baddeley AD, Hitch GJ, \& Allen RJ (2009). Working memory and binding in sentence recall. Journal of Memory and Language, 61(3), 438-456.

Baldo JV, \& Dronkers NF (2006). The role of inferior parietal and inferior frontal cortex in working memory. Neuropsychology, 20(5), 529. [PubMed: 16938015]

Baldo JV, Klostermann EC, \& Dronkers NF (2008). It's either a cook or a baker: Patients with conduction aphasia get the gist but lose the trace. Brain and Language, 105(2), 134-140. [PubMed: 18243294]

Baldo JV, Katseff S, \& Dronkers NF (2012). Brain regions underlying repetition and auditory-verbal short-term memory deficits in aphasia: Evidence from voxel-based lesion symptom mapping. Aphasiology, 26(3-4), 338-354. [PubMed: 24976669]

Bayles KA, Tomoeda CK, \& Rein JA (1996). Phrase repetition in Alzheimer's disease: Effect of meaning and length. Brain and Language, 54(2), 246-261. [PubMed: 8811956]

Bonner MF, Ash S, \& Grossman M (2010). The new classification of primary progressive aphasia into semantic, logopenic, or nonfluent/agrammatic variants. Current Neurology and Neuroscience Reports, 10(6), 484-490. [PubMed: 20809401]

Buchsbaum BR, Baldo J, Okada K, Berman KF, Dronkers NF, D’Esposito M, et al. (2011). Conduction aphasia, sensory-motor integration, and phonological short-term memory - an aggregate analysis of lesion and fMRI data. Brain and Language, 119, 119-128. [PubMed: 21256582] 
Carter JV, Pan J, Rai SN, \& Galandiuk S (2016). ROC-ing along: Evaluation and interpretation of receiver operating characteristic curves. Surgery, 159(6), 1638-1645. [PubMed: 26962006]

Conrad R, \& Hull AJ (1964). Information, acoustic confusion and memory span. British Journal of Psychology, 55(4), 429-432. [PubMed: 14237884]

Dahnke R, Yotter RA, \& Gaser C (2013). Cortical thickness and central surface estimation. Neuroimage, 65, 336-348. [PubMed: 23041529]

Damasio H, \& Damasio AR (1980). The anatomical basis of conduction aphasia. Brain,103(2), 337350. [PubMed: 7397481]

Davies M (2008). The corpus of contemporary American English: 450 million words, 1990-present. http://corpus.byu.edu/coca/.

Dronkers NF (2000). The pursuit of brain-language relationships. Brain and Language,71(1), 59-61. [PubMed: 10716807]

Dronkers NF, Redfern BB, \& Ludy C (1998). Brain regions associated with conduction aphasia and echoic rehearsal. Journal of the International Neuropsychological Society, 4, 23-24.

Dunn LM, \& Dunn LM (1981). Manual for the peabody picture vocabulary test-revised. Circle Pines, MN: American Guidance Service.

Folstein MF, Folstein SE, \& McHugh PR (1975). "Mini-mental state”: A practical method for grading the cognitive state of patients for the clinician. Journal of Psychiatric Research, 12(3), 189-198. [PubMed: 1202204]

Friederici AD (2012). The cortical language circuit: From auditory perception to sentence comprehension. Trends in Cognitive Sciences, 16, 262-326. [PubMed: 22516238]

Galantucci S, Tartaglia MC, Wilson SM, Henry ML, Filippi M, Agosta F, ... Gorno-Tempini ML (2011). White matter damage in primary progressive aphasias: A diffusion tensor tractography study. Brain, 134(10), 3011-3029. [PubMed: 21666264]

Geldmacher DS, Quigg M, \& Elias WJ (2007). MR tractography depicting damage to the arcuate fasciculus in a patient with conduction aphasia. Neurology, 69(3), 321-322. [PubMed: 17636077]

Goodglass H, Kaplan E, \& Weintraub S (1983). Boston naming test. Lea \& Febiger.

Gorno-Tempini ML, Dronkers NF, Rankin KP, Ogar JM, Phengrasamy L, Rosen HJ, ... Miller BL (2004). Cognition and anatomy in three variants of primary progressive aphasia. Annals of Neurology, 55(3), 335-346. [PubMed: 14991811]

Gorno-Tempini ML, Brambati SM, Ginex V, Ogar J, Dronkers NF, Marcone A, ... Miller BL (2008). The logopenic/phonological variant of primary progressive aphasia. Neurology, 71(16), 1227 1234. [PubMed: 18633132]

Gorno-Tempini ML, Hillis AE, Weintraub S, Kertesz A, Mendez M, Cappa SEEA, ... Manes F (2011). Classification of primary progressive aphasia and its variants. Neurology, 76(11), 1006-1014. [PubMed: 21325651]

Green E, \& Howes DH (1977). The nature of conduction aphasia: A study of anatomic and clinical features and of underlying mechanisms. Studies in Neurolinguistics, 3, 123-156.

Henry ML, \& Gorno-Tempini ML (2010). The logopenic variant of primary progressive aphasia. Current Opinion in Neurology, 23(6), 633. [PubMed: 20852419]

Hickok G, \& Poeppel D (2007). The cortical organization of speech processing. Nature Reviews Neuroscience, 8, 393-402. [PubMed: 17431404]

Hickok G, Okada K, \& Serences JT (2009). Area Spt in the human planum temporale supports sensory-motor integration for speech processing. Journal of Neurophysiology, 101(5), 2725-2732. [PubMed: 19225172]

Hickok G (2009). The functional neuroanatomy of language. Physics of Life Reviews, 6(3),121-143. [PubMed: 20161054]

Hickok G (2012). Computational neuroanatomy of speech production. Nature Reviews Neuroscience, 13, 135-145. [PubMed: 22218206]

Hodges JR, \& Miller B (2001). The neuropsychology of frontal variant frontotemporal dementia and semantic dementia. Introduction to the special topic papers: Part II. Neurocase, 7(2), 113-121. [PubMed: 11320159] 
Hodges JR, \& Patterson K (1996). Nonfluent progressive aphasia and semantic dementia: A comparative neuropsychological study. Journal of the International Neuropsychological Society, 2(6), 511-524. [PubMed: 9375155]

Hu WT, McMillan C, Libon D, Leight S, Forman M, Lee VY, ... Grossman M (2010). Multimodal predictors for Alzheimer disease in nonfluent primary progressive aphasia. Neurology, 75(7), 595602. [PubMed: 20713948]

Jacquemot C, \& Scott SK (2006). What is the relationship between phonological short-term memory and speech processing? Trends Cognitive Sciences, 10, 480-486. [PubMed: 16997610]

Kertesz A (1982) Western aphasia battery. New York Grune and Stratton.

Knott R, Patterson K, \& Hodges JR (1997). Lexical and semantic binding effects in short-term memory: Evidence from semantic dementia. Cognitive Neuropsychology, 14(8), 1165-1216.

Kreisler A, Godefroy O, Delmaire C, Debachy B, Leclercq M, Pruvo JP, \& Leys D (2000). The anatomy of aphasia revisited. Neurology, 54(5), 1117-1123. [PubMed: 10720284]

Leyton CE, Savage S, Irish M, Schubert S, Piguet O, \& Ballard KJ (2014). Verbal repetition in primary progressive aphasia and Alzheimer's disease. Journal of Alzheimer's Disease, 41(2), 575-585.

Martin RC (1987). Articulatory and phonological deficits in short-term memory and their relation to syntactic processing. Brain and Language, 32(1), 159-192. [PubMed: 3651806]

Martin RC, Shelton JR, \& Yaffee LS (1994). Language processing and working memory: Neuropsychological evidence for separate phonological and semantic capacities. Journal of Memory and Language, 33(1), 83-111.

McCarthy RA, \& Warrington EK (1987). The double dissociation of short-term memory for lists and sentences: Evidence from aphasia. Brain, 110(6), 1545-1563. [PubMed: 3427400]

Majerus S (2013). Language repetition and short-term memory: An integrative framework. Frontiers in Human Neuroscience, 7, 357. [PubMed: 23874280]

Meltzer JA, Rose NS, Deschamps T, Leigh RC, Panamsky L, Silberberg A, ... Links KA (2016). Semantic and phonological contributions to short-term repetition and long-term cued sentence recall. Memory \& Cognition, 44(2), 307-329. [PubMed: 26374330]

Mesulam M (1982). Slowly progressive aphasia without generalized dementia. Annals of Neurology, 11(6), 592-598. [PubMed: 7114808]

Mesulam M, Wieneke C, Rogalski E, Cobia D, Thompson C, \& Weintraub S (2009).Quantitative template for subtyping primary progressive aphasia. Archives of Neurology, 66(12), 1545-1551. [PubMed: 20008661]

Mesulam MM, Wieneke C, Thompson C, Rogalski E, \& Weintraub S (2012).Quantitative classification of primary progressive aphasia at early and mild impairment stages. Brain, 135(5), 1537-1553. [PubMed: 22525158]

Meyer AM, Snider SF, Campbell RE, \& Friedman RB (2015). Phonological short-term memory in logopenic variant primary progressive aphasia and mild Alzheimer's disease. Cortex, 71, 183-189. [PubMed: 26232551]

Neary D, Snowden JS, Gustafson L, Passant U, Stuss D, Black SASA, ... Boone K (1998). Frontotemporal lobar degeneration: A consensus on clinical diagnostic criteria. Neurology, 51(6), 1546-1554. [PubMed: 9855500]

Ogar JM, Dronkers NF, Brambati SM, Miller BL, \& Gorno-Tempini ML (2007). Progressive nonfluent aphasia and its characteristic motor speech deficits. Alzheimer Disease \& Associated Disorders, 21(4), S23-S30. [PubMed: 18090419]

Rogalski E, Cobia D, Harrison TM, Wieneke C, Thompson CK, Weintraub S,et al. (2011). Anatomy of language impairments in primary progressive aphasia. Journal of Neuroscience, 31, 3344-3350. [PubMed: 21368046]

Rohrer JD, Ridgway GR, Crutch SJ, Hailstone J, Goll JC, Clarkson MJ, ... Warrington EK (2010). Progressive logopenic/phonological aphasia: Erosion of the language network. Neuroimage, 49(1), 984-993. [PubMed: 19679189]

Shallice T, \& Warrington EK (1977). Auditory-verbal short-term memory impairment and conduction aphasia. Brain and Language, 4(4), 479-491. [PubMed: 922463]

Shuren JE, Schefft BK, Yeh HS, Privitera MD, Cahill WT, \& Houston W (1995). Repetition and the arcuate fasciculus. Journal of Neurology, 242(9), 596-598. [PubMed: 8551322] 
Tanabe H, Sawada T, Inoue N, Ogawa M, Kuriyama Y, \& Shiraishi J (1987). Conduction aphasia and arcuate fasciculus. Acta Neurologica Scandinavica, 76(6), 422-427. [PubMed: 3434200]

Weintraub S, Rubin NP, \& Mesulam MM (1990). Primary progressive aphasia: Longitudinal course, neuropsychological profile, and language features. Archives of Neurology, 47(12), 1329-1335. [PubMed: 2252450]

Whitwell JL, Jones DT, Duffy JR, Strand EA, Machulda MM, Przybelski SA, ... Petersen RC (2015). Working memory and language network dysfunctions in logopenic aphasia: A task-free fMRI comparison with Alzheimer's dementia. Neurobiology of Aging, 36(3), 1245-1252. [PubMed: 25592958]

Yamada K, Nagakane Y, Mizuno T, Hosomi A, Nakagawa M, \& Nishimura T (2007). MR tractography depicting damage to the arcuate fasciculus in a patient with conduction aphasia. Neurology, 68(10) 789-789. [PubMed: 17339591]

Yotter RA, Nenadic I, Ziegler G, Thompson PM, \& Gaser C (2011). Local cortical surface complexity maps from spherical harmonic reconstructions. NeuroImage, 56(3), 961-973. [PubMed: 21315159] 


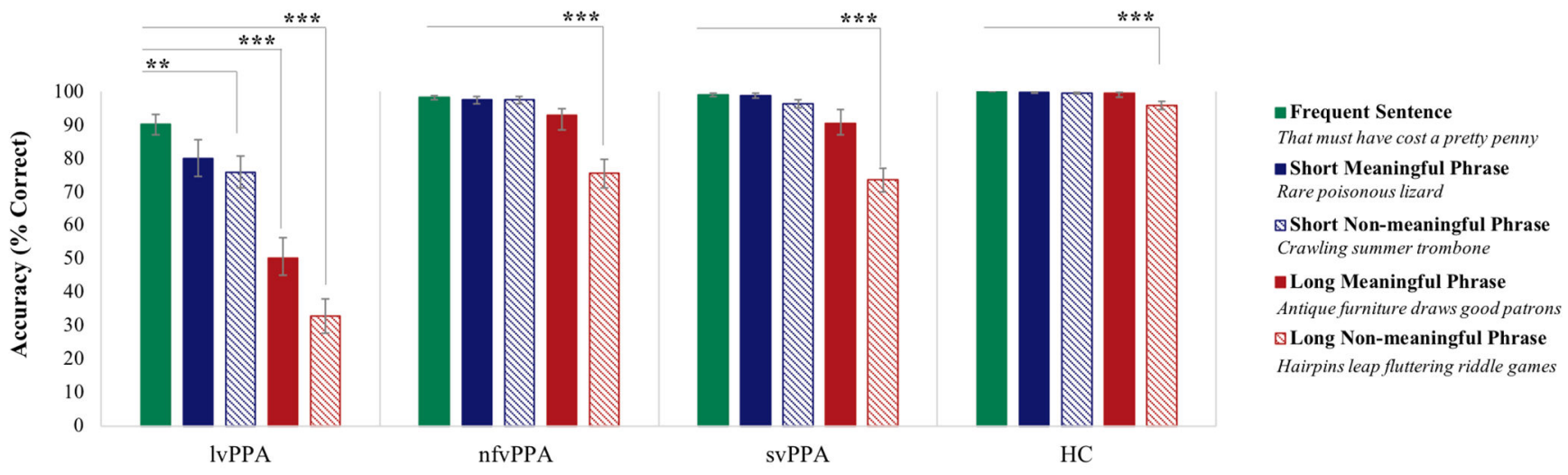

Fig. 1.

The repetition performance distribution for the group of HC and the three PPA variants. Mean accuracy (\% correct) representing the number of correctly repeated phrases for the three PPA variants and the group of HC. Asterisks indicate significantly impaired performances across conditions at $\mathrm{p}<0.05(*), \mathrm{p}<0.01\left({ }^{* *}\right)$, and $\mathrm{p}<0.001\left(^{* * *}\right)$. 
A. Group by Length interaction

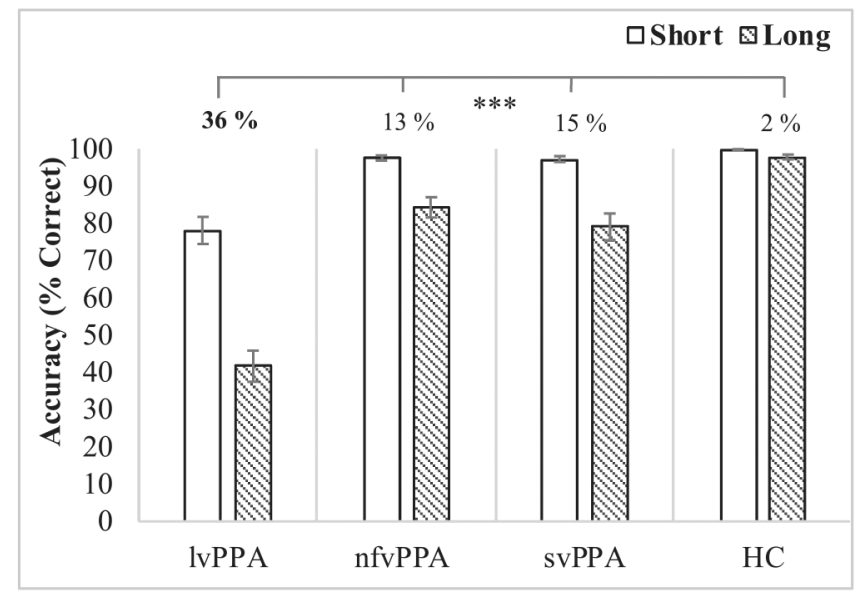

B. Group by Meaning interaction

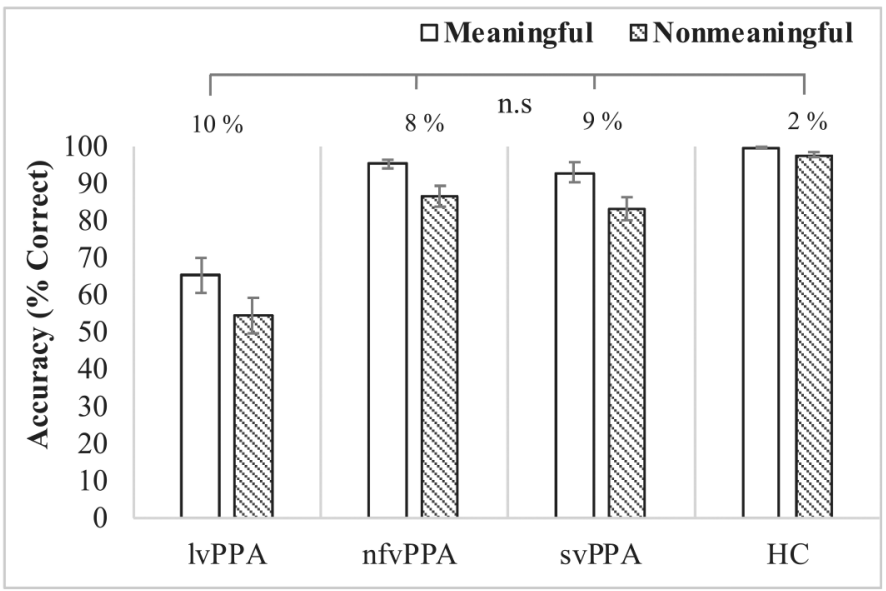

\section{Fig. 2.}

Performance on the repetition test across conditions for groups of $\mathrm{HC}$ and the three PPA variants. (A) Mean accuracy (\% correct) for the two length conditions, and (B) Mean accuracy (\% correct) for the two meaning conditions; significant two-way interactions at $\mathrm{p}<$ $\left.0.001{ }^{* * *}\right) ; \%$ differences between short and long, and meaningful and non-meaningful conditions for each group are presented above each bar of the graph. 


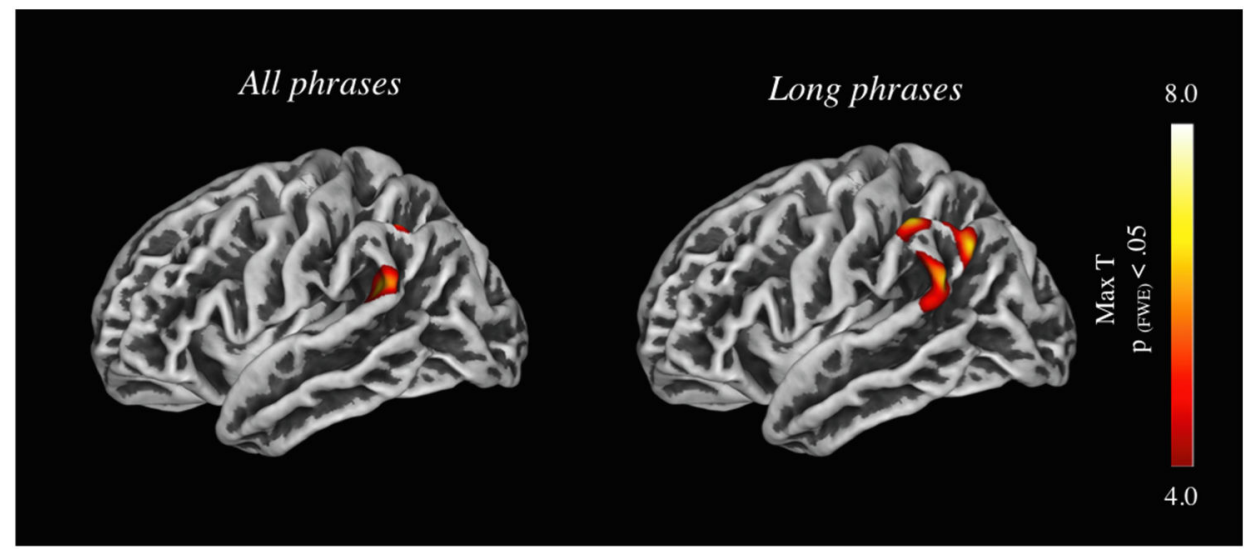

Fig. 3.

Cortical thickness maps showing areas where cortical thinning was associated with repetition performances on all phrases, and long (over short) phrases across PPAs. The presented map is thresholded at $\mathrm{p}<0.05$ family-wise error (FWE) corrected both at peaklevel and cluster extent based thresholding. Covariates: age, gender, handedness, and severity (measured by the Clinical Dementia Rating box score). 

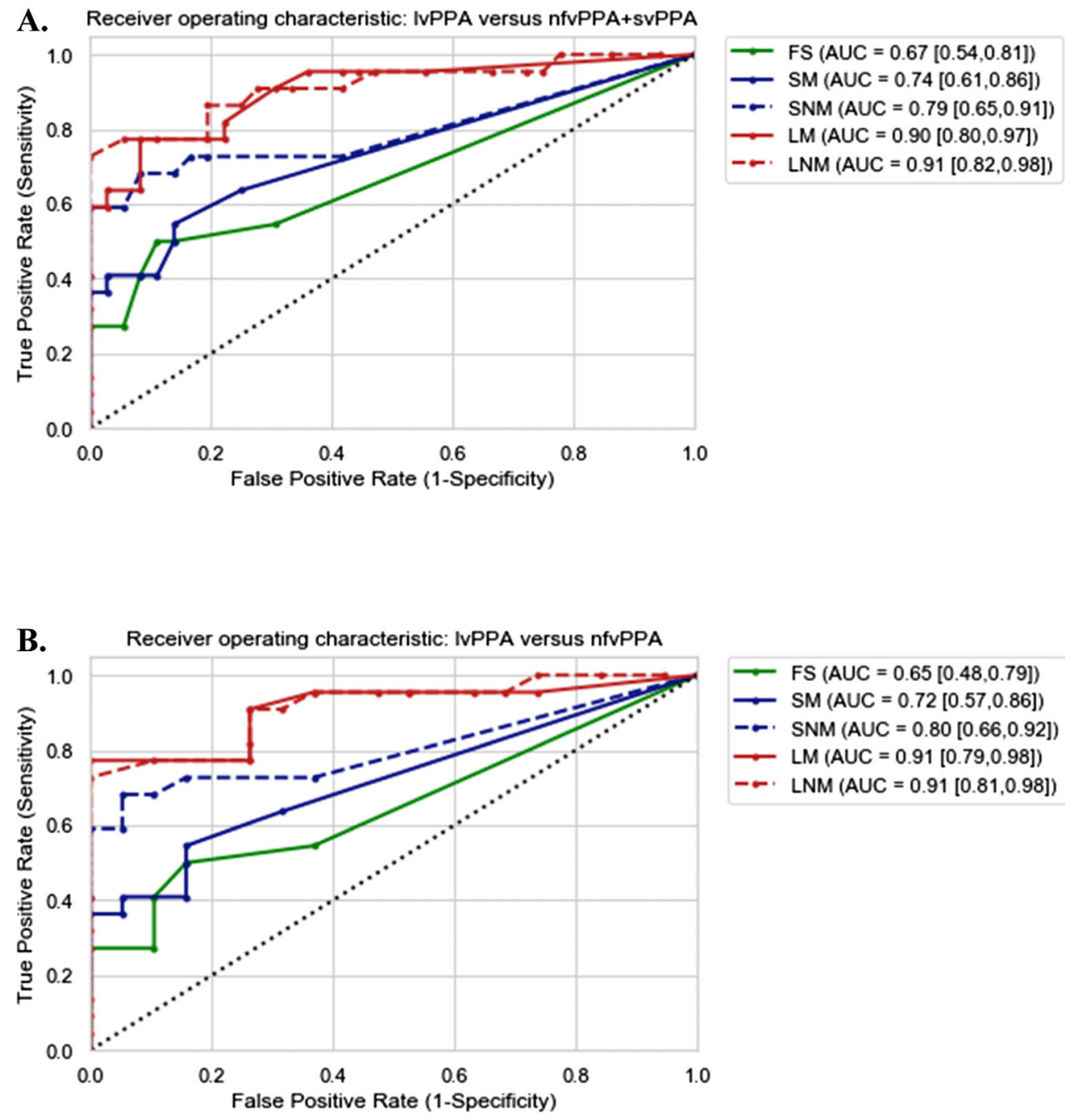

Fig. 4.

Receiver Operating Characteristics (ROC) curves display the capacity of different subscores on the repetition test to discriminate lvPPA from the non-logopenic patients (A), and nfvPPA (B). The ROC curves of Frequent Sentences (FS; green solid line), Short Meaningful (SM; blue solid line) and Short Non-Meaningful (SNM; blue dashed line), and Long Meaningful (LM; red solid line) and Long Non-Meaningful (LNM; red dashed line) phrases. The area under the curve (AUC) and Confidence Interval (CI) values are reported for each condition showing the overall ability of different sub-scores to correctly identify lvPPA from the nfvPPA and svPPA. A value of 1.0 is a perfect test, $0.9-0.99$ is an excellent test, $0.8-0.89$ is a good test, $0.7-0.79$ is a fair test, $0.51-0.69$ is a poor test, and 0.5 is of no value; A prediction model is considered strong with a values of $>0.8$ (Carter, Pan, Rai, \& Galandiuk, 2016). 


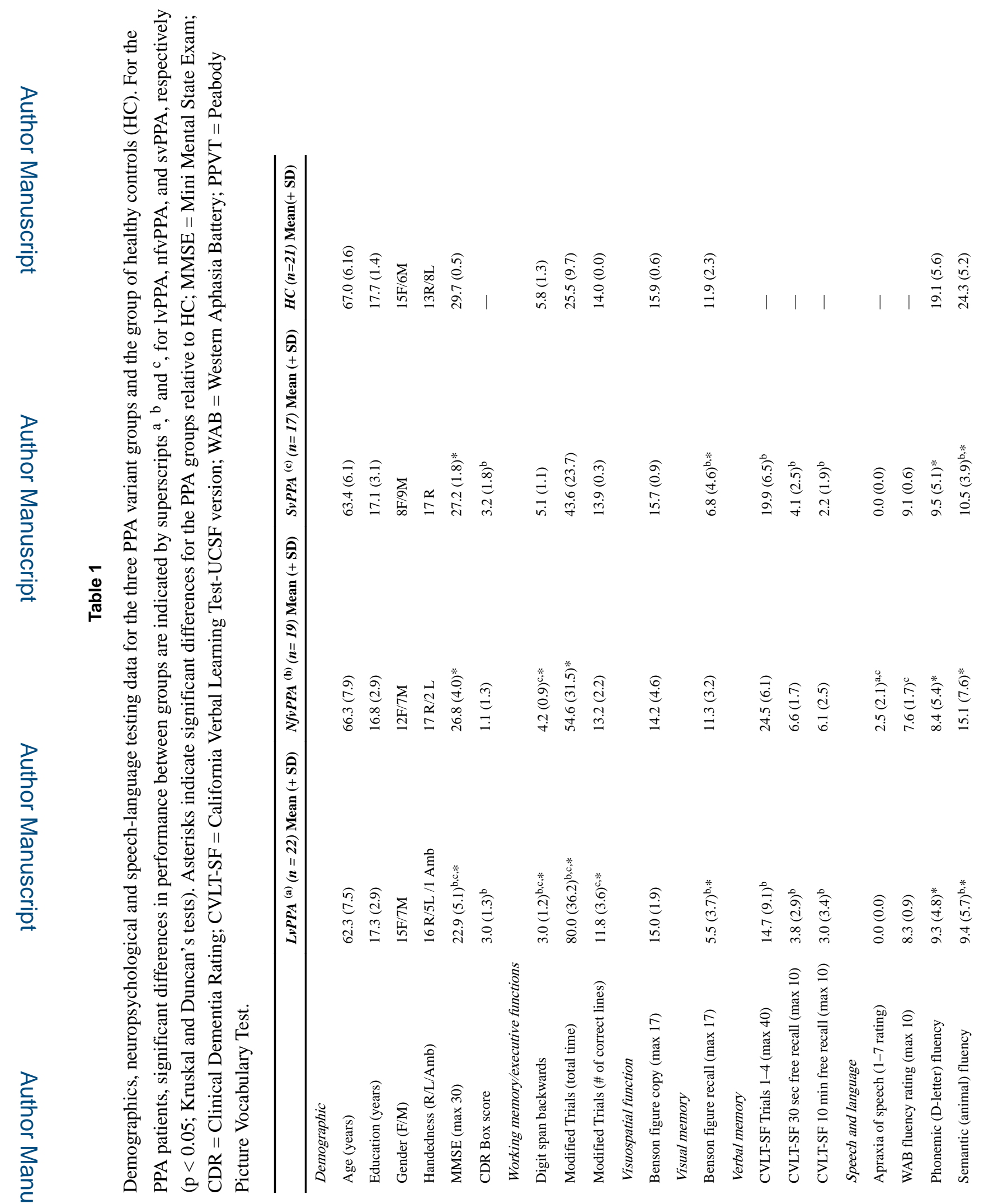

Brain Lang. Author manuscript; available in PMC 2019 July 31. 
Lukic et al.

Page 24

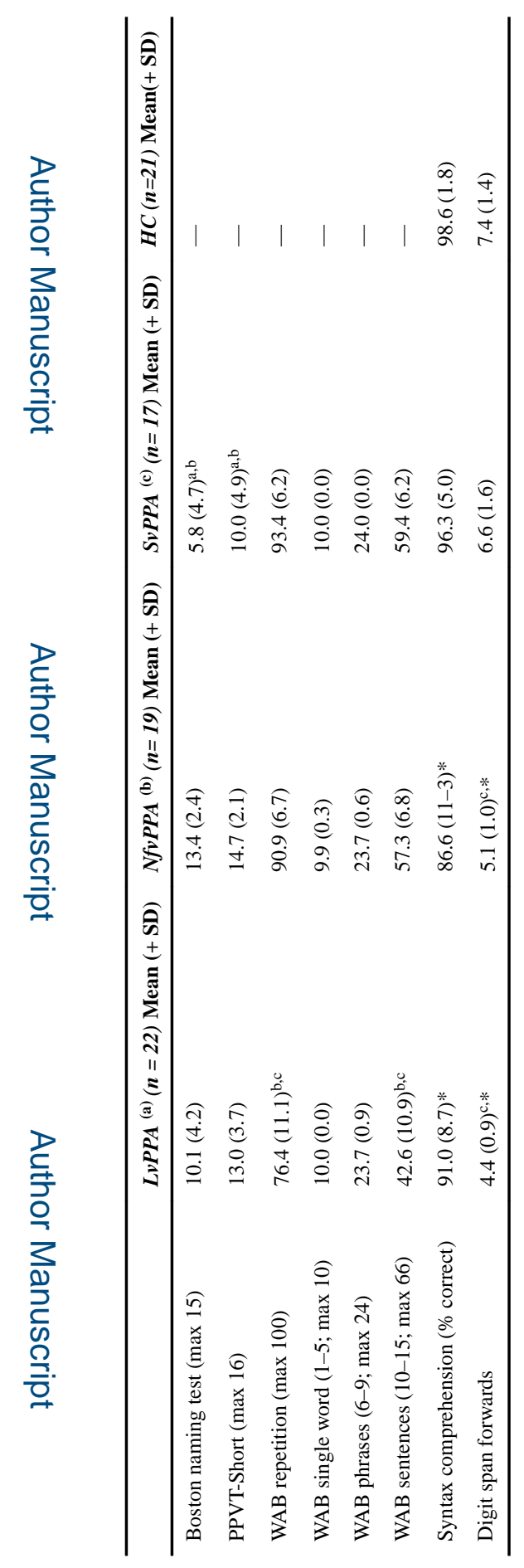

로을

Brain Lang. Author manuscript; available in PMC 2019 July 31. 


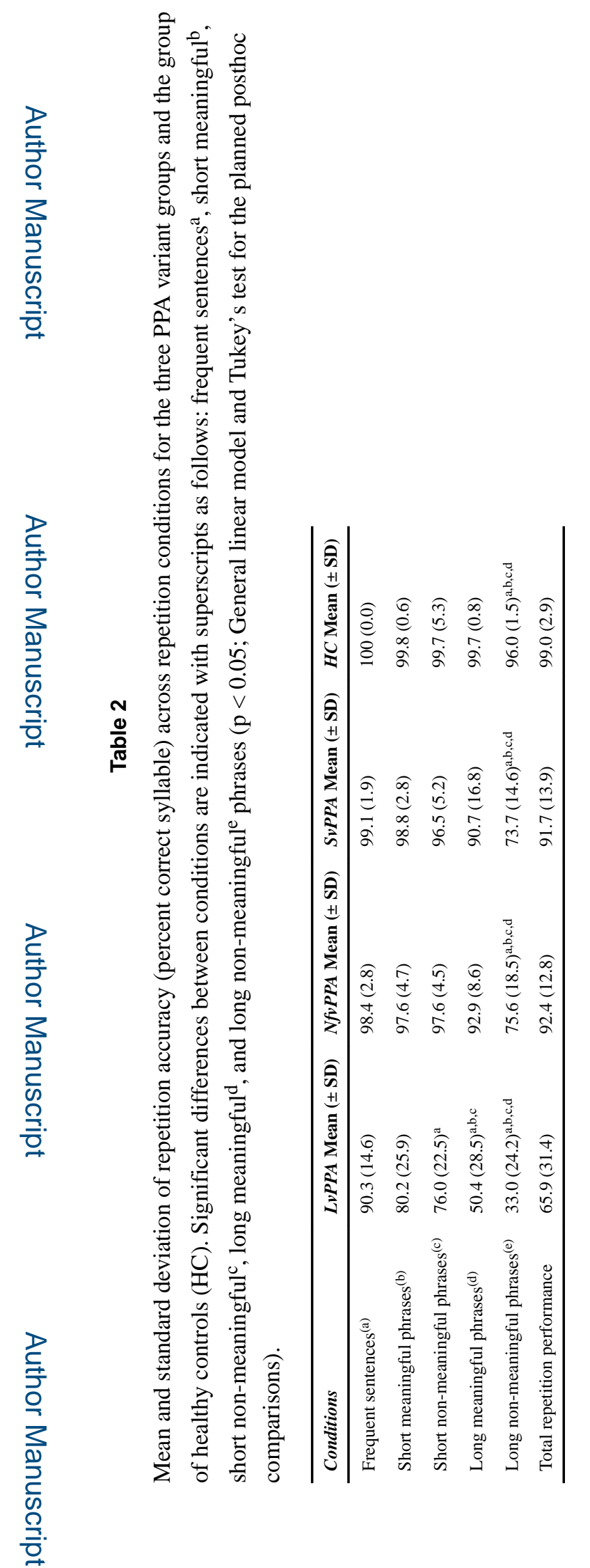

Brain Lang. Author manuscript; available in PMC 2019 July 31. 


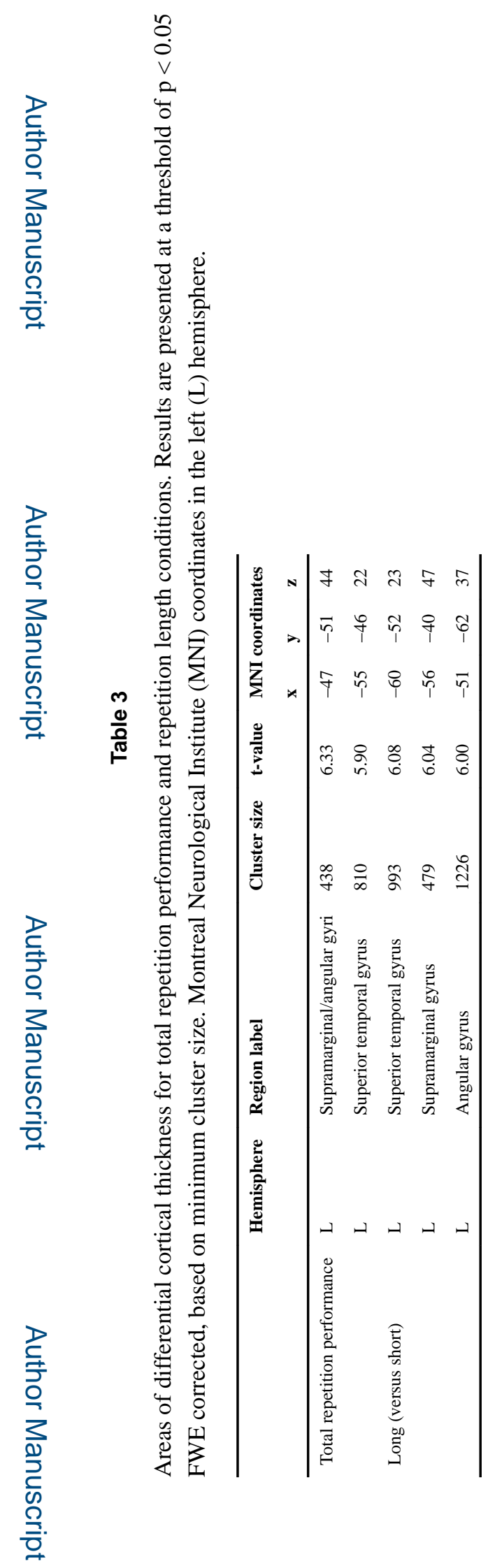

Brain Lang. Author manuscript; available in PMC 2019 July 31. 